\title{
Treatment of Active Crohn's Disease with Exclusive and Partial Enteral Nutrition: A Pilot Study in Adults
}

\author{
Catherine L. Wall ${ }^{\mathrm{a}}$ Richard B. Gearry ${ }^{\mathrm{b}}$ Andrew S. Day ${ }^{\mathrm{a}}$ \\ a Department of Paediatrics, University of Otago Christchurch, Christchurch, New Zealand; \\ ${ }^{b}$ Department of Medicine, University of Otago Christchurch, Christchurch, New Zealand
}

\section{Keywords}

Crohn's disease - Enteral nutrition - Faecal calprotectin ·

Insulin-like growth factor-1

\begin{abstract}
Background and Aim: Enteral nutrition (EN) is not commonly used for the treatment of adults with active Crohn's disease (CD), despite patient interest in nutrition-based alternatives to corticosteroids and evidence of efficacy in paediatric $\mathrm{CD}$. The aim of this study was to assess the impact of 2 different EN regimens on disease symptoms, nutrition and inflammatory markers in young adults with active CD. Methods: A prospective non-randomized pilot study of adults aged 1640 years with active $C D$ on endoscopy or imaging was undertaken. Patients were sequentially recruited to use 2 weeks of exclusive EN (EEN) followed by either 6 weeks of EEN or partial EN (PEN) with usual diet. Assessments of disease symptoms, nutrition and inflammatory markers were undertaken at baseline and throughout the treatment. Results: Thirtyeight patients with active disease were recruited. Thirty-two (84\%) patients completed 2 weeks of EEN and had significant improvements in disease symptoms $(p=0.003)$, serum c-reactive protein (CRP; $p=0.005)$, insulin-like growth factor-1
\end{abstract}

\section{KARGER}

() 2018 S. Karger AG, Basel

E-Mail karger@karger.com

www.karger.com/iid $(p=0.006)$ and faecal calprotectin ( $F C ; p=0.028)$. During the following 6 weeks, 21 patients continued EEN (14 [67\%] completed treatment) and 11 patients used PEN (9 [82\%] completed treatment). Initial improvements in symptoms, CRP and nutrition markers were sustained over the next 6 weeks on both treatments. FC non-significantly increased in 5 out of 9 patients who used PEN and at week 8 FC was greater than $500 \mu \mathrm{g} / \mathrm{g}$ in 9 out of 14 and 7 out of 9 patients who used exclusive or PEN respectively. There was no significant difference in clinical outcomes between the 2 groups at week 8 . Conclusion: Two weeks of EEN significantly improved disease symptoms, nutrition and inflammatory markers. Further treatment with exclusive or PEN maintained initial improvements.

(c) 2018 S. Karger AG, Basel

\section{Introduction}

Crohn's disease (CD), an incurable inflammatory bowel disease, can develop at any age [1]. Intestinal inflammation may present clinically as abdominal pain, increased frequency of loose bowel motions, and/or biochemically with elevated serum and fecal inflammatory 
markers [2]. There are many pharmaceutical and surgical treatments for active CD $[3,4]$. Nutrition-based treatments, the most common being exclusive enteral nutrition (EEN), is recommended as a first-line therapy to treat active paediatric CD [5]. EEN is now commonly used to treat active $\mathrm{CD}$ in children and adolescents in New Zealand (NZ) [6], Australia [7], Asia [8], Canada [9] and Europe [10]. Adults with CD are interested in nutrition-based alternatives to corticosteroids [11], and EEN is regularly used in Japan [12], and increasingly in China [13] to treat adults with active CD. For adults living in Western countries, EEN is not currently recommended as a first-line treatment for active CD $[2,14]$.

Previous studies of EEN to induce CD remission in adult patients have provided variable results [15]. One of the reasons cited for the variability in outcomes is the poor palatability of nutrition formulas and poorer adherence to the exclusive regimens [15]. Partial EN (PEN) may help alleviate issues with adherence to EEN: this approach has been trailed in children and adolescents, again with variable results [16-18].

In the paediatric age group, EEN offers patients benefits over and above the induction of disease remission, including high rates of mucosal healing, which are not achieved with corticosteroids [19]. Faecal calprotectin (FC) has been suggested as a biomarker of mucosal healing due to its moderate correlation with endoscopic assessments of mucosal healing $[20,21]$. A few paediatric studies have reported changes in FC following EEN treatment [16, 22-24], but no studies in adult cohorts have reported changes in FC consequent to EEN.

This study aimed first to investigate the feasibility and effectiveness of EEN and a novel PEN regimen in young adults with active $\mathrm{CD}$ on symptoms of active disease and second to document the impact of these treatments on nutrition and inflammatory markers including FC.

\section{Materials and Methods}

\section{Participants}

From May 2013 to December 2015, young adults with active CD aged 16-40 years old were invited at the discretion of the consulting gastroenterologist, to choose between nutrition therapy or corticosteroids to treat active disease. Eligible patients had CD involving at least the ileum and were managed by gastroenterologists in Christchurch, NZ. Patients were excluded from the study if they had isolated colonic disease, active psychological illness or had taken corticosteroids in the last fortnight. Concomitant use of other CD medications did not limit eligibility; however, the use of corticosteroid medications was not permitted and patients on mesalazine, biological or thiopurine medications needed to be on an ex- isting and stable dose and despite medication use still have active CD. Patients could be started on maintenance of remission dose of thiopurine medication once established and after 4 weeks of Enteral nutrition (EN) treatment. Active disease was defined as active disease visible by endoscopy or radiology or an elevated FC. Eligible patients were referred to 1 registered dietitian who obtained their informed consent and managed their nutrition therapy treatment. Ethical approval was given by the NZ Northern B Health and Disability Ethics Committee (ethics reference 13/NTB/11) and the pilot clinical trial was registered with Australia New Zealand Clinical Trial Registry (trial number 363665).

\section{Enteral Nutrition Treatments}

Patients were sequentially recruited to use 2 weeks of EEN followed by another 6 weeks of EEN or PEN. Patients recruited between May 2013 and February 2015 were offered only EEN and patients recruited from March to December 2015 were offered only EEN followed by PEN. Patients' nutrition requirements were calculated using bioimpedance analysis basal metabolic rate multiplied by a physical activity factor [25]. Patients' nutritional requirements were reviewed fortnightly according to weight change and appetite.

EEN treatment required patients to drink multiple cartons $(200 \mathrm{~mL})$ of a polymeric $6.32 \mathrm{~kJ} / \mathrm{mL}(1.5 \mathrm{kcal} / \mathrm{mL})$ oral nutritional formula (Ensure Plus, Abbott Laboratories, The Netherlands) daily. In addition to the prescribed EN, patients were encouraged to drink additional fluids either as water and/or black unsweetened tea, coffee or herbal tea and avoid all other foods and fluids. EEN was initiated by gradually replacing meals and snacks with EN over a period of 3 days.

PEN treatment comprised of EN plus 1 small meal per day (lunch or dinner) of solid food. Lunch and dinner were chosen because these meals are more likely to contain larger amounts of protein and vegetable fibre than breakfast and these meals are commonly shared with friends and/or family. The sharing of food with friends and family was an important aspect because, during the EEN intervention of this study, many patients anecdotally reported EEN to be socially isolating. Patients were encouraged to eat a balanced meal similar to their usual eating habits. After both 8 -week treatments, patients reintroduced usual foods and fluids and reduced EN intake over a period of 3 days.

\section{Assessments}

Patients were assessed by the dietitian at weeks $0,2,4,6$, and 8 . At baseline, demographics, family history, disease phenotype and outcome data were collected. Assessments at all 5 study appointments included a serum inflammatory marker (c-reactive protein [CRP]), serum nutrition markers (insulin-like growth factor-1 [IGF-1]) and albumin, anthropometrics (body mass index [BMI]), intestinal inflammation biomarker (FC) and clinical disease activity (Harvey Bradshaw Index [HBI]).

Serum CRP, albumin and IGF-1 were measured by Canterbury Health Laboratories, NZ using immunoturbidimetry, bromocresol purple assay kit (Abbott $\mathrm{C}$ series analyser) and the iSYS automated chemiluminescence immunoassay (Immunodiagnostic Systems, United Kingdom) respectively. FC was batch analyzed from stool stored at $-80^{\circ} \mathrm{C}$ using a commercial enzyme linked immunosorbent assay kit (BÜHLMANN fCAL, EK-CAL2, Switzerland) as per the manufacturer's instructions. The kit test range was $60-3,600 \mu \mathrm{g} / \mathrm{g}$. An elevated HBI was not an 
inclusion criterion to receive EN therapy, and therefore improvements in symptom/activity scores rather than rates of disease remission were calculated.

Dietary intake of any food or fluids was self-reported fortnightly. Patients using PEN were asked to provide examples of the meals that they had consumed for their 1 meal per day. Significant and continued deviations from the protocols resulted in withdrawal from the study. During EEN, non-habitual and small amounts of usual foods/fluids were assumed to have a negligible impact on average energy intake. Nutrient and energy intake during PEN was calculated from a 3-day food record using a smart phone/tablet app Evernote ${ }^{\odot}$. Patients recorded their intake of food and fluids in real time with photographs of the meal and any leftovers alongside text descriptions. This electronic food diary has been validated against a paper food diary in young children (unpublished data) but not in adult patients. Nutrient analysis of food records was completed using "Kai-culator" dietary assessment software (version 1.15 c Department of Human Nutrition, University of Otago, NZ).

\section{Statistical Analysis}

The results are presented as percentage of responses, medians and ranges. Many of the variables were not normally distributed; therefore, groups were compared using Mann-Whitney U test, Wilcoxon matched-pairs signed rank test, Fisher's exact test and chi-square test. Statistical significance was present with $p<0.05$. Statistical tests and graphs were prepared in Prism 6 version 6.05 (GraphPad Software Inc.).

\section{Results}

\section{Baseline Characteristics of the Treatment Groups}

Thirty-eight patients were referred for EN therapy. The first 25 patients were offered 2 weeks of EEN therapy followed by a further 6 weeks EEN and the following 13 patients were offered 2 weeks of EEN followed by 6 weeks of PEN. The 2 groups were similar at baseline with the exception of serum albumin, which was significantly lower in the PEN group (Table 1).

Patients who had a history of weight loss prior to starting EN treatment ( $n=19)$ had a median IGF-1 standard deviation score (SDS), which was significantly lower $(-1.00$ SDS compared with 0.10 SDS, $p=0.01)$ than the median score of patients $(n=19)$ whose weight was stable prior to treatment. There was no difference $(p=$ $0.588)$ in the median BMI or serum albumin $(p=0.239)$ of those who had, and had not, experienced recent weight loss.

Concurrent use of medication, except corticosteroids, was permitted. Eight patients were established on stable doses of medications at baseline but still had active disease (Table 1). No patients were started on mesalazine or biological medications during EN treatment.

\section{Adherence to Treatments}

Figure 1 summarises the flow of patients through the study. Thirty-eight patients started EEN but within a few days of starting, 2 patients elected to use corticosteroids instead of EEN and another 4 patients did not tolerate the EN formula (increased diarrhoea or nausea) and therefore did not successfully initiate EEN. After the first 2 weeks of treatment, 21 patients continued on EEN treatment. During the following 6 weeks, 7 patients did not complete EEN treatment: 1 needed surgery for a small bowel perforation, one reintroduced usual diet at week 5 in response to work stress, 1 developed nausea, 1 deviated significantly from the protocol, 1 did not respond after 4 weeks, 1 had persistent diarrhoea, which resolved after stopping the formula and 1 needed nasogastric tube feeding to meet nutritional requirements and when the tube split opted not to have another tube placed. After the initial 2 weeks of EEN, 11 patients moved onto PEN treatment of which 2 patients did not complete the treatment: 1 patient, who had a small bowel perforation and was waiting for surgery, flared after introducing usual food and returned to EEN until the surgery could be performed, and 1 patient had not responded by week 4 and was changed to corticosteroid treatment.

There was no significant difference in the proportion of patients who did not complete EEN compared with PEN treatment $(p=0.502)$. However, more adolescent patients $(<18$ years old) did not complete either EEN or PEN treatment than those aged over 18 years $(8 / 15$ compared with $3 / 23, p=0.012$ ).

\section{Dietary Intake}

All patients, except 1, reported 100\% compliance with the EEN protocol during the first fortnight of treatment. Thereafter, self-reported intake of usual foods and fluids in the EEN group was minimal, as was intake of foods and fluids apart from the 1 small meal during PEN treatment. Individuals' total energy intake 6,276-13,807 kJ/day (1,500-3,300 kcal/day), percentage of estimated energy requirements (\% EER) and energy intake per weight varied widely (Table 2) as did the amount of physical activity. The 1 meal per day during PEN typically consisted of a protein food (red meat, chicken, eggs or fish) with a carbohydrate food (bread, rice, potato, sweet potato or pasta) and cooked or raw vegetables.

\section{Clinical Outcomes}

During the first 2 weeks of EEN $(n=32)$ median HBI fell from 5 to 3 points $(p=0.003)$, median serum CRP fell from 10 to $5 \mathrm{mg} / \mathrm{L}(p=0.005)$, median FC fell from 927 
Table 1. Baseline characteristics of patients referred for enteral nutrition therapy

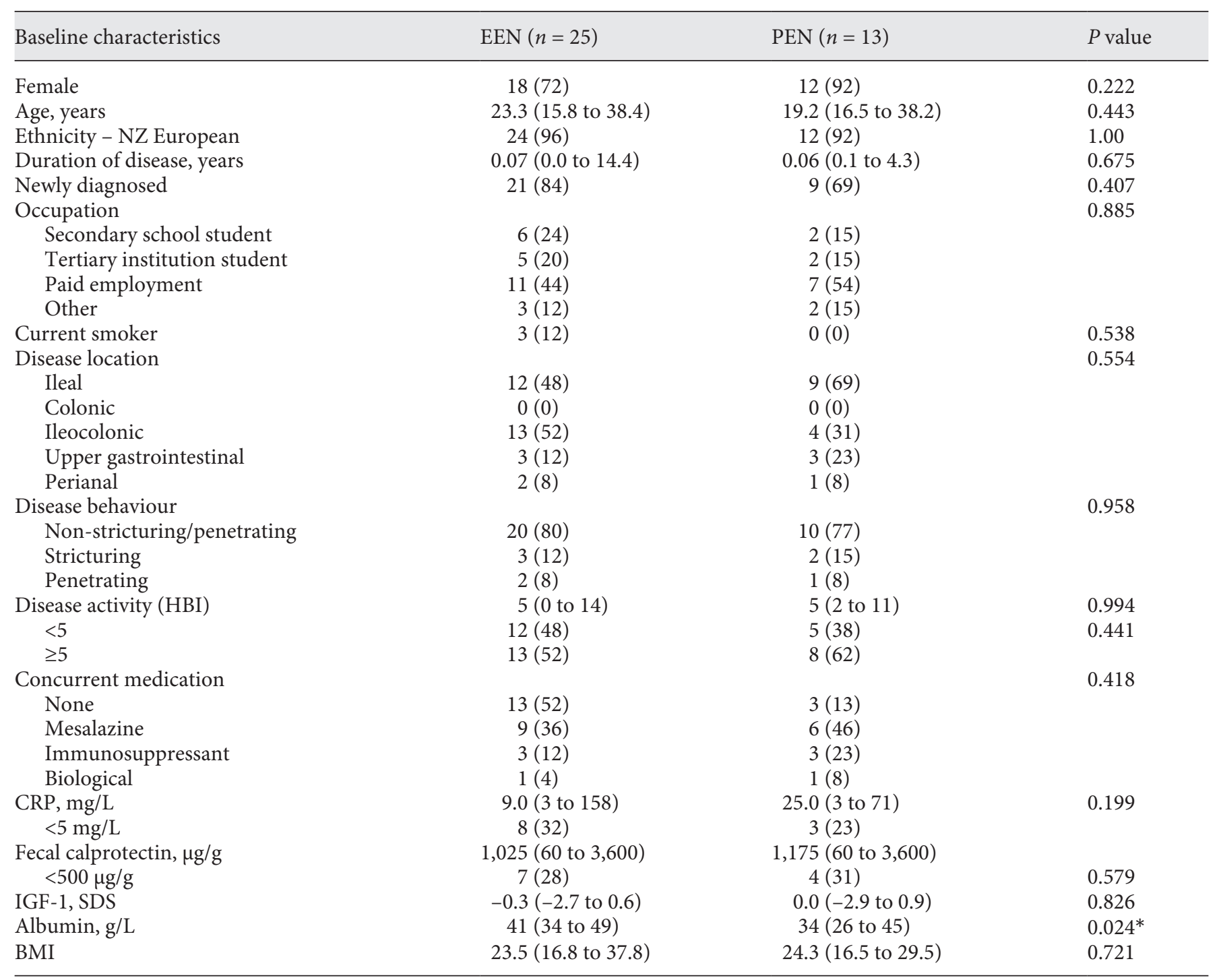

Values are median (range) or $n(\%)$.

$* p<0.05$. Treatment groups were compared using either Mann-Whitney test, Fisher's exact test or chi-square test.

EEN, exclusive enteral nutrition; HBI, Harvey Bradshaw index; IGF-1, insulin-like growth factor-1; PEN, partial enteral nutrition; SDS, standard deviation score.

to $674 \mu \mathrm{g} / \mathrm{g}(p=0.028)$, median IGF-1 SDS improved from 0.0 to $0.05(p=0.006)$ and median serum albumin was unchanged at $39.5 \mathrm{~g} / \mathrm{L}$ (Fig. 2).

Fourteen patients used EEN for another 6 weeks after the initial 2 weeks of EEN. The improvements in inflammatory markers observed during the first 2 weeks were sustained to week 8 and there was further improvement in the median HBI $(p=0.031)$. At week 8, 5/14 (36\%) patients had an FC $<500 \mu \mathrm{g} / \mathrm{g}$ compared with 4 out of 14
(29\%) at baseline. Patients using EEN lost weight during treatment, the median BMI fell from 23.7 to $23.3 \mathrm{~kg} / \mathrm{m}^{2}$ $(\mathrm{W}=-79, p=0.01)$ although the minimum BMI increased from 18.5 to 19.8 and nutrition markers serum IGF-1 and albumin improved during EEN treatment (Fig. 3).

Nine patients used PEN after an initial 2 weeks of EEN. The median HBI, CRP and FC remained stable after PEN treatment although FC increased in 5 out of 9 
Table 2. Average total energy intake during the first and last 2 weeks of enteral nutrition therapy

Fig. 1. Flowchart of patients treated with exclusive and PEN. EEN, exclusive enteral nutrition; EN, enteral nutrition; PEN, partial enteral nutrition.

\begin{tabular}{lcc}
\hline & EEN, median (range) & PEN, median (range) \\
\hline At week 2, $n$ & 21 & 11 \\
\% EER & $93(64-117)$ & $90(69-129)$ \\
$\mathrm{kcal} / \mathrm{kg} /$ day & $36.5(23.9-51.2)$ & $34.2(28.7-40.5)$ \\
$\mathrm{kcal} \mathrm{from} \mathrm{EN}$ & $2,100(1,350-3,150)$ & $1,950(1,350-2,550)$ \\
At week 8, $n$ & 14 & $8^{+}$ \\
\% EER & $93(64-114)$ & $80(61-124)$ \\
$\mathrm{kcal} / \mathrm{kg} /$ day & $30.9(24.3-51.5)$ & $28.2(20.8-44.5)$ \\
$\%$ TE from food & $\mathrm{n} / \mathrm{a}$ & $35.5(14.7-59.9)^{+}$ \\
$\mathrm{kcal}$ from EN & $2,050(1,350-3,200)$ & $1,050(750-1,800)^{\S}$ \\
\hline
\end{tabular}

${ }^{+} n=8$ because one patient did not upload their electronic food record, ${ }^{\S} n=9$.

$\%$ EER, percentage of estimated energy requirements; EN, enteral nutrition; EEN, exclusive enteral nutrition; n/a, not applicable; PEN, partial enteral nutrition; TE, total energy.

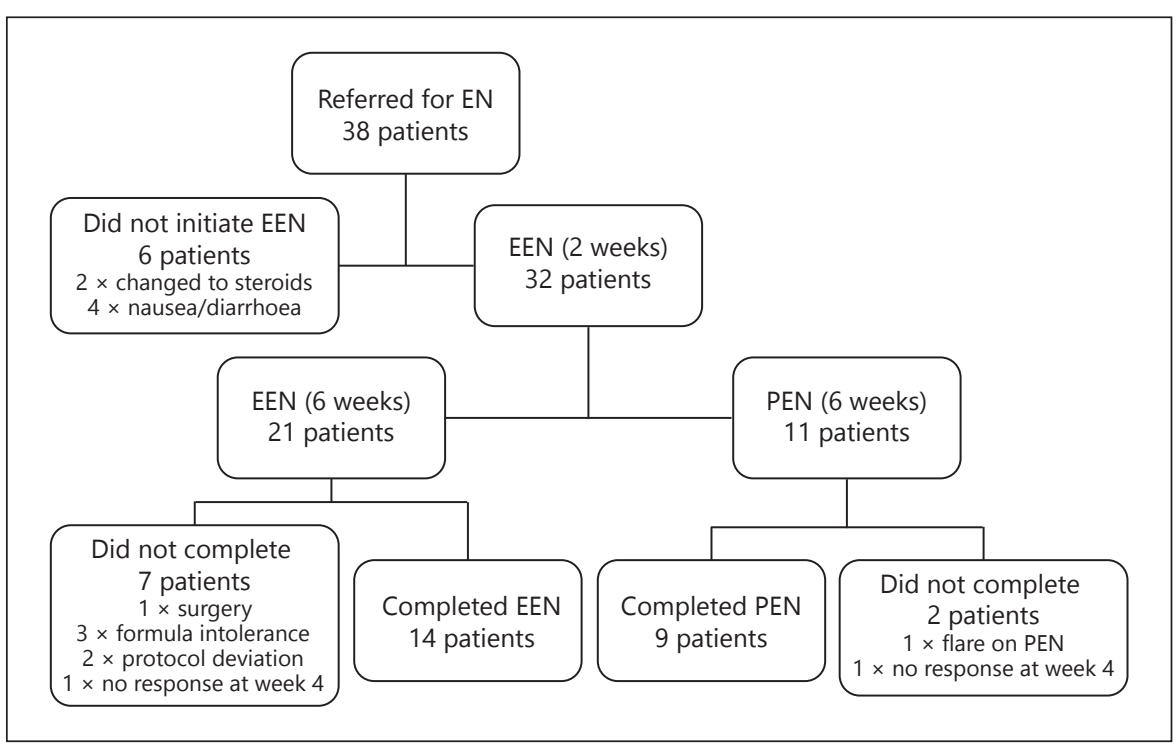

patients during PEN treatment (Fig. 2) and 2 out of 9 (22\%) had an FC less than $500 \mu \mathrm{g} / \mathrm{g}$ compared with 3 out of $9(33 \%)$ of patients at baseline. Clinical outcomes in the 9 PEN patients were not correlated with volume of EN consumed or the percentage of total energy from solid food (data not shown). Patients using PEN had a minimal change in median BMI from 25.2 to 24.7 ( $p>$ 0.05 ) and the minimum BMI increased from 16.5 to 18.5.

There were no significant differences in disease activity, nutrition or inflammatory markers at week 8 between patients who used EEN for 8 weeks compared with patients who used 2 weeks of EEN followed by 6 weeks of PEN.

\section{Discussion}

This prospective non-randomized pilot clinical trial of EEN and PEN is one of the first PEN studies to include only adults with active CD. This study used a novel PEN regimen, which included 2 weeks of EEN followed by 6 weeks of PEN with 1 small meal of usual food. Three studies have been published, which used PEN to treat active CD. One of these studies of PEN, which did not include an EEN control group, found that PEN with a specific food exclusion diet effectively induced disease remission in $70 \%$ of children and adults [18]. Whereas, 2 of the studies found that EEN was superior to PEN with a free diet $[16,17]$. In contrast, this study found that PEN with 
Fig. 2. Clinical parameters at baseline and after 2 weeks EEN treatment $(n=33)$. CRP, c-reactive protein; EEN, exclusive enteral nutrition; IGF-1, insulin-like growth factor-1, HBI, Harvey Bradshaw index.
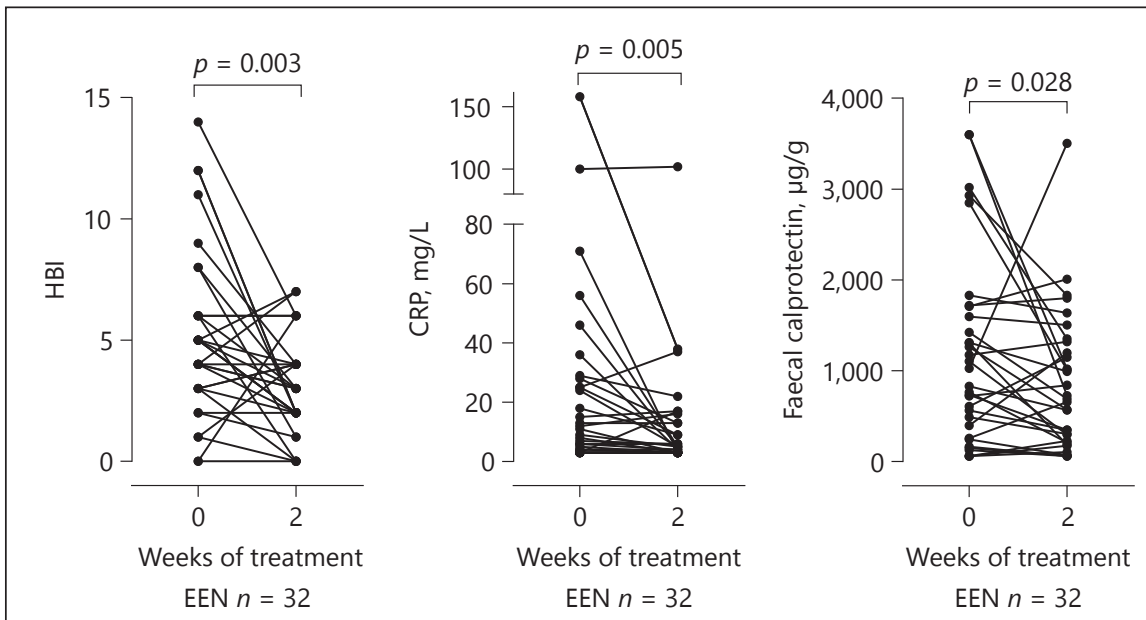

EEN $n=32$
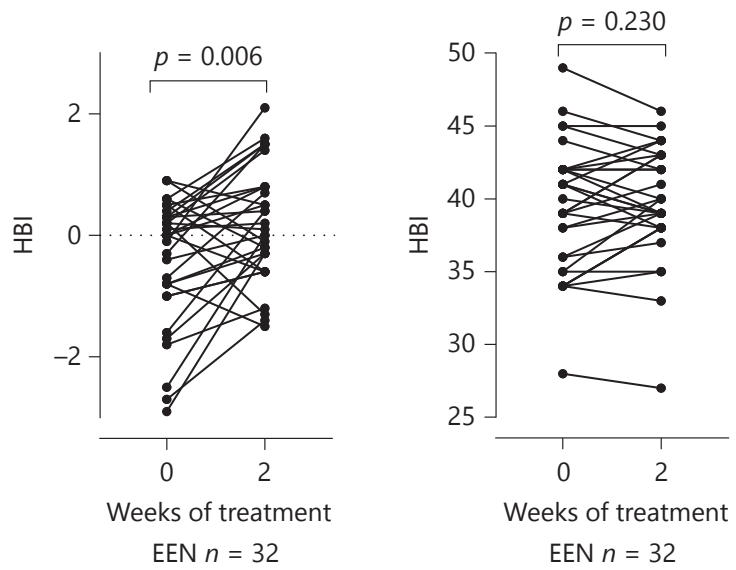

usual food resulted in similar outcomes to EEN at week 8 . The lack of difference between the PEN and EEN treatments may be due to the use of 2 weeks of EEN prior to the reintroduction of usual food. During the first 2 weeks of EEN, patients had significant improvements in disease activity and inflammatory markers, such early improvements have been observed previously in the adult EEN literature $[13,26,27]$. Further, there is some evidence that patients with newly diagnosed disease respond better to nutrition therapy than patients with long-standing intestinal inflammation [28]. The inclusion of mostly newly diagnosed patients and those with mild disease symptoms may have contributed to the comparable outcomes between the 2 treatments.

Serum IGF-1 is a marker of nutrition status and has been suggested as a marker of disease activity $[29,30]$ due to its reduced expression in the presence of pro-inflammatory cytokines [31]. An early rise in IGF-1 subsequent to EEN treatment has previously been documented in paediatric IBD studies [30,32] but has not been reported in adults with CD. Both paediatric studies concluded that early improvements in IGF-1 concentration were due to reduced inflammation rather than purely an improvement in nutrition intake. In this study, median serum IGF-1 concentrations increased significantly after 2 weeks of treatment despite suboptimal caloric intake and reductions in BMI, and corresponded with a reduction in serum CRP and FC. These results support the paediatric IBD observations that IGF- 1 is more than just a marker of nutrition status and that improvements in serum IGF1 concentration may also reflect reduced inflammation.

FC has been suggested as a reliable non-invasive measure of endoscopic disease activity [20, 21, 33, 34], but cut-off concentrations to distinguish active from inactive inflammation remain controversial [20]. Some paediatric studies have reported improvements in FC following EEN [22-24] but to date there are no reports of FC changes consequent to EEN or PEN in adult cohorts. The current study found a non-significant trend towards improved FC after 2 weeks of EEN, but further treatment 


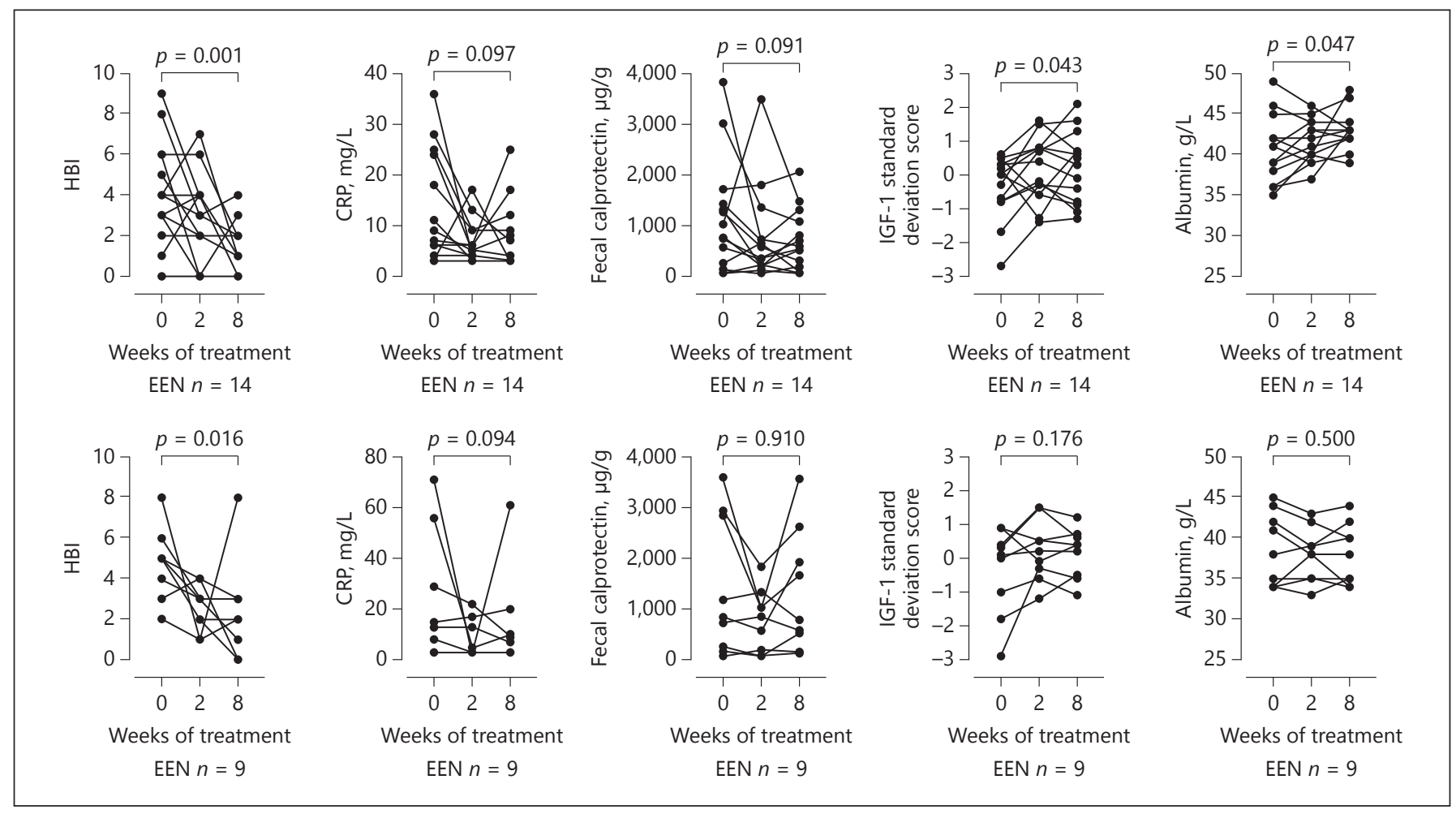

Fig. 3. Clinical parameters at baseline, week 2 and week 8 of treatment of patients treated only with EEN or with 2 weeks of exclusive followed by 6 weeks of PEN. CRP, c-reactive protein;
EEN, exclusive enteral nutrition; IGF-1, insulin-like growth factor-1; PEN, partial enteral nutrition, HBI, Harvey Bradshaw index. with EEN did not result in further improvement in FC concentration. Paediatric EEN studies have also observed that FC often remains elevated post EEN treatment [22, $24,35]$. One PEN study has reported FC. It observed that $14 \%$ children treated with PEN had an FC less than $250 \mu \mathrm{g} / \mathrm{g}$ compared with $45 \%$ treated with EEN and $62 \%$ treated with a biologic medication [16]. Their results, along with those presented here, suggest that the inclusion of usual food with EN may limit gut mucosal healing.

Patient withdrawal from EN treatment is common in many of the published adult studies and was previously associated with poor palatability of the nutrition formula [15]. This study used a more palatable polymeric formula and as a result no patient withdrew from the treatment due to the unpalatable formula. However, non-completion of treatment was higher than anticipated. Most $(78 \%)$ of the study cohort were female and 30\% were less than 18 years old. It has previously been suggested that female adolescents with IBD find EN therapy more attractive than corticosteroid treatment, due to the potential negative impact of CS treatment on body image [36]. The high proportion of female participants may represent a selection bias be- cause patients were referred to the study at the discretion of the consulting gastroenterologist rather than systematic referral of all patients meeting the study inclusion criteria. The high proportion of female patients and secondary school age patients is likely to have affected treatment completion. Females and older adolescents are more likely to withdraw from treatment due to non-adherence [10] and this trend was observed in the current study, whereby $73 \%$ of adolescents did not complete EN treatment compared with only $26 \%$ of young adults aged 18 years and older. EN treatment may be a more acceptable treatment for patients who have finished secondary education and moved into the workforce or tertiary education.

A limitation of this study may be the lack of a non-EN control group. Previous randomized controlled trials of EEN and corticosteroids in paediatric patients have shown the 2 treatments have comparable efficacy $[15,37]$, whereas intention to treat analysis in adult cohorts have found corticosteroids to be superior to $\operatorname{EEN~}[26,38,39]$; however, per protocol, outcomes may be more similar [15]. The aim of this study was not to repeat previous research but to assess the feasibility and effectiveness of 
EEN and a combined EEN and PEN regimen. These pilot study results cannot be compared directly with the current standard treatment for mild to moderate adult CD, but the outcomes are of interest to patients and clinicians involved in trialing nutrition-based treatments.

No single CD treatment is effective for all patients; likewise, nutrition-based therapies are unlikely to be appropriate for all patients either. For patients who are interested in using a nutritional approach, or who want to avoid using corticosteroids, EEN and PEN therapies are a feasible treatment option. In these modest cohorts of young adults with active $\mathrm{CD}$, the data indicates that EEN effectively reduces clinical symptoms and markers of inflammation within the first 2 weeks of treatment. Further investigation into the potential role of a PEN regimen after an initial period of EEN is warranted; however, the composition of the food included in the regimen needs further investigation as does the impact of solid food on FC and mucosal healing. EN therapy is a feasible and effective option to treat active CD in young adults who have finished secondary education and could be offered to patients interested in using a nutritional therapy approach.

\section{Acknowledgements}

This work was supported by the grants from the NZ Society of Gastroenterology, Dietitians NZ, Australasian Society of Parenteral and Enteral Nutrition, NZ Federation of Graduate Women and The Maurice and Phyllis Paykel Trust.

\section{Disclosure Statement}

The authors have no conflicts of interest to disclose.

\section{References}

1 Su H, Gupta HSV, Day AS, Gearry RB: Rising incidence of inflammatory bowel disease in Canterbury, New Zealand. Inflamm Bowel Dis 2016;22:2238-2244.

2 Gomollon F, Dignass A, Annese V, Tilg H, Van Assche G, Lindsay J, et al: 3rd European evidence-based consensus on the diagnosis and management of Crohn's disease 2016: part 1: diagnosis and medical management. J Crohns Colitis 2017;11:3-25

3 Bernstein CN, Eliakim A, Fedail S, Fried M, Gearry R, Goh K, et al: World Gastroenterology Organisation Global Guidelines Inflammatory Bowel Disease: World Gastroenterology Organisation, 2015. http://www.worldgastroenterology.org/guidelines/ global-guidelines/inflammatory-bowel-disease-ibd/inflammatory-bowel-disease-ibdenglish.

4 Mayberry JF, Lobo A, Ford AC, Thomas A: NICE clinical guideline (CG152): the management of Crohn's disease in adults, children and young people. Aliment Pharmacol Ther 2013;37:195-203.

5 Ruemmele FM, Veres G, Kolho KL, Griffiths A, Levine A, Escher JC, et al: Consensus guidelines of ECCO/ESPGHAN on the medical management of pediatric Crohn's disease. J Crohns Colitis 2014;8:1179-1207.

6 Wall C, Day A, Gearry R: Dietitian experience with exclusive enteral nutrition for the treatment of Crohn disease. J Nutr Med Diet Care 2016;2:015.

7 Day AS, Stephenson T, Stewart M, Otley AR: Exclusive enteral nutrition for children with Crohn's disease: use in Australia and attitudes of Australian paediatric gastroenterologists. J Paediatr Child Health 2009;45:337-341.
8 Hida N, Nakamura S, Hahm KB, Sollano JD, Zhu Q, Rani AA, et al: A questionnaire-based survey on the diagnosis and management of inflammatory bowel disease in East Asian countries in 2012. Digestion 2014;89:88-103.

9 Stewart M, Day AS, Otley A: Physician attitudes and practices of enteral nutrition as primary treatment of paediatric Crohn disease in North America. J Pediatr Gastroenterol Nutr 2011;52:38-42.

10 de Bie C, Kindermann A, Escher J: Use of exclusive enteral nutrition in paediatric Crohn's disease in The Netherlands. J Crohns Colitis 2013;7:263-270.

11 Wall CL, Gearry RB, Day AS: Polymeric formula is more palatable than elemental formula to adults with Crohn's disease. e-SPEN J 2014;9:e200-e203.

12 Matsui T, Sakurai T, Yao T: Nutritional therapy for Crohn's disease in Japan. J Gastroenterol 2005;40(suppl 16):25-31.

13 Guo Z, Wu R, Zhu W, Gong J, Zhang W, Li Y, et al: Effect of exclusive enteral nutrition on health-related quality of life for adults with active Crohn's disease. Nutr Clin Pract 2013; 28:499-505.

14 Dignass A, Van Assche G, Leman M, Soderholm J, Colombel JF, Danese S, et al: The second European evidence-based consensus on the diagnosis and management of Crohn's disease: current management. J Crohns Colitis 2010;4:28-62.

15 Wall CL, Day AS, Gearry RB. Use of exclusive enteral nutrition in adults with Crohn's disease: a review. World J Gastroenterol 2013;19: 7652-7660.

16 Lee D, Baldassano RN, Otley AR, Albenberg L, Griffiths AM, Compher C, et al: Compara- tive effectiveness of nutritional and biological therapy in North American children with active Crohn's disease. Inflamm Bowel Dis 2015;21:1786-1793.

17 Johnson T, Macdonald S, Hill SM, Thomas A Murphy MS: Treatment of active Crohn's disease in children using partial enteral nutrition with liquid formula: a randomised controlled trial. Gut 2006;55:356-361.

18 Sigall-Boneh R, Pfeffer-Gik T, Segal I, Zangen T, Boaz M, Levine A: Partial enteral nutrition with a Crohn's disease exclusion diet is effective for induction of remission in children and young adults with Crohn's disease. Inflamm Bowel Dis 2014;20:1353-1360.

19 Borrelli O, Cordischi L, Cirulli M, Paganelli M, Labalestra V, Uccini S, et al: Polymeric diet alone versus corticosteroids in the treatment of active pediatric Crohn's disease: a randomized controlled open-label trial. Clin Gastroenterol Hepatol 2006;4:744-753.

20 Falvey JD, Hoskin T, Meijer B, Ashcroft A, Walmsley R, Day AS, et al: Disease activity assessment in IBD: clinical indices and biomarkers fail to predict endoscopic remission. Inflamm Bowel Dis 2015;21:824831

21 Goutorbe F, Goutte M, Minet-Quinard R, Boucher AL, Pereira B, Bommelaer G, et al: Endoscopic factors influencing fecal calprotectin value in Crohn's disease. J Crohns Colitis 2015;9:1113-1119.

22 Grogan JL, Casson DH, Terry A, Burdge GC, El-Matary W, Dalzell AM: Enteral feeding therapy for newly diagnosed pediatric crohn's disease: a double-blind randomized controlled trial with two years follow-up. Inflamm Bowel Dis 2012;18:246-253. 
23 Navas López VM, Blasco-Alonso J, Lacasa Maseri S, Girón Fernández-Crehuet F, Serrano Nieto MJ, Vicioso Recio MI: Exclusive enteral nutrition continues to be first line therapy for pediatric Crohn's disease in the era of biologics. An Pediatr (Barc) 2015;83: 47-54.

24 Gerasimidis $\mathrm{K}$, McGrogan P, Edwards CA: The aetiology and impact of malnutrition in paediatric inflammatory bowel disease. J Hum Nutr Diet 2011;24:313-326.

25 Schofield WN: Predicting basal metabolic rate, new standards and review of previous work. Hum Nutr Clin Nutr 1985;39(suppl 1): 5-41.

26 Lochs H, Steinhardt HJ, Klaus-wentz B, Zeitz M, Vogelsang H, Sommer H, et al: Comparison of enteral nutrition and drug treatment in active Crohn's disease. Results of the European Cooperative Crohn's disease Study IV. Gastroenterology 1991;101:881-888.

27 Verma S, Brown S, Kirkwood B, Giaffer MH: Polymeric versus elemental diet as primary treatment in active Crohn's disease: a randomized, double-blind trial. Am J Gastroenterol 2000;95:735-739.

28 Day AS, Whitten KE, Lemberg DA, Clarkson C, Vitug-Sales M, Jackson R, et al: Exclusive enteral feeding as primary therapy for Crohn's disease in Australian children and adoles- cents: a feasible and effective approach. J Gastroenterol Hepatol 2006;21:1609-1614.

29 Alempijevic T, Jovanovic I, Popovic D, Kovacevic N, Milutinnoiv AS, Krstic M: IGF-1 as a marker of disease activity and nutritional status in patients with inflammatory bowel disease. Indian J Gastroenterol 2008;27:247.

30 Bannerjee K, Camacho-Hubner C, Babinska K, Dryhurst KM, Edwards R, Savage MO, et al: Anti-inflammatory and growth-stimulating effects precede nutritional restitution during enteral feeding in Crohn disease. JPGN 2004;38:270-275.

31 O'Connor JC, McCusker RH, Strle K, Johnson RW, Dantzer R, Kelley KW: Regulation of IGF-I function by proinflammatory cytokines: at the interface of immunology and endocrinology. Cell Immunol 2008;252:91-110.

32 Beattie RM, Camacho-Hubner C, Wacharasindhu S, Cotterill AM, Walker-Smith JA, Savage MO: Responsiveness of IGF-I and IGFBP-3 to therapeutic intervention in children and adolescents with Crohn's disease. Clin Endocrinol (Oxf) 1998;49:483489.

33 D'Inca R, Dal Pont E, Di Leo V, Ferronato A, Fries W, Vettorato MG, et al: Calprotectin and lactoferrin in the assessment of intestinal inflammation and organic disease. Int J Colorectal Dis 2007;22:429-437.
34 Boon GJ, Day AS, Mulder CJ, Gearry RB: Are faecal markers good indicators of mucosal healing in inflammatory bowel disease? World J Gastroenterol 2015;21:11469-11480.

35 Frivolt K, Schwerd T, Werkstetter KJ, Schwarzer A, Schatz SB, Bufler P, et al: Repeated exclusive enteral nutrition in the treatment of paediatric Crohn's disease: predictors of efficacy and outcome. Aliment Pharmacol Ther 2014;39:1398-1407.

36 Goodhand J, Hedin CR, Croft NM, Lindsay JO: Adolescents with IBD: the importance of structured transition care. J Crohns Colitis 2011;5:509-519.

37 Heuschkel RB, Menache CC, Megerian JT, Baird AE: Enteral nutrition and corticosteroids in the treatment of acute Crohn's disease in children. J Pediatr Gastroenterol Nutr 2000;31:8-15.

38 Malchow H, Steinhardt HJ, Lorenzmeyer H, Strohm WD, Rasmussen S, Sommer H, et al: Feasibility and effectiveness of a defined-formula diet regimen in treating active Crohn's disease. European Cooperative Crohn's Disease Study III. Scand J Gastroenterol 1990;25:235-244.

39 Gorard DA, Hunt JB, Paynejames JJ, Palmer KR, Rees RGP, Clark ML, et al: Initial response and subsequent course of Crohn's disease treated with elemental diet or prednisolone. Gut 1993;34:1198-1202. 